Aus dem neurobiologischen Laboratorium der Universität Berlin.

\title{
Über die nervösen Endorgane im häutigen Labyrinth der Säugetiere.
}

\author{
Von \\ Max Bielschowsky und Gustav Brühl. \\ Hierzu Tafel $\mathrm{V}$ und VI.
}

\section{Einleitung.}

Für die Erforschung des Zusammenhanges von Sinneszellen und Nervenfasern sind die Maculae und Cristae acusticae seit langer Zeit ein Lieblingsobjekt der Histologen. Der Grund dafür liegt darin, dass die grobkalibrigen Verzweigungen des Vestibularnerven auf ihren marklosen Endstrecken relativ leicht darzustellen und über weite Strecken zu verfolgen sind; und dazu kommt, dass die reizaufnehmenden Haarzellen hier zu einem Epithelverbande von sehr einfachem Gefïge angeordnet sind. Trotz dieser günstigen topographischen Verhältnisse ist auch heute das Problem, wie Zelle und Faser miteinander verkettet sind, noch nicht vollkommen gelöst, was um so auffälliger erscheinen mag, als eine grosse Zahl hervorragender Forscher sich mit diesem Thema beschäftigt hat. Die Differenzen in den Anschauungen der verschiedenen Antoren sind im wesentlichen durch die Methoden begründet, mit denen sie gearbeitet haben, und nirgends kann man den Einfluss der Methodik auf histologische Vorstellungen klarer demonstrieren als hier. Die klassischen Bearbeiter des Gehörorgans, welche die Nervenendstellen einer genauen Beobachtung unterzogen, Hasse, Boettcher, Waldeyer und Retzius vertraten wohl vorwiegend auf Grund von Isolationspräparaten und einfacheren Färbungen die Anschauung, dass die Nervenfasern im Protoplasma der Haarzellen endigen, dass also Faser und Zelle organisch zu einem Ganzen miteinander verschmolzen sind.

Diese Vorstellung wurde verlassen, als die Golgische Methode ihren Siegeslauf begann und mit scharfen Kontrasten die schwarzen Nervenelemente auf farblosem oder hellem Grunde 
zeigte. Die Mehrzahl der Autoren glaubte jetzt zu sehen, dass die Vestibularisfasern an den Haarzellen frei endigten, also nur durch Kontakt mit ihnen in Beziehung standen (Ka is er, ${ }^{1}$ ) v. Lenhossék, ${ }^{2}$ ) van Gehuchten ${ }^{3}$ ) und Ramón y Cajal ${ }^{4}$ ). Bezüglich der Form der freien Endigungen wichen die Angaben der Autoren allerdings nicht unerheblich voneinander $a b$; in dem prinzipiell wichtigsten Punkte stimmten sie aber überein, dass nämlich Faser und Zelle sich gegenseitig nur oberflächlich berühren. Auch der um die Histologie des Ohres so hochverdiente Retzi u s ${ }^{5}$ ) verliess unter dem Einfluss der Chromsilberpräparate seinen alten Standpunkt und verkündete als einer der ersten den Satz, dass die Endigung der Acusticusfasern stets unter dem Bilde frei auslaufender Verästelungen erfolgte. Zu ganz ähnlichen Anschauungen. führten auch die Bilder der fast $z u$ derselben Zeit von Ehrlich gefundenen vitalen Methylenblaufärbung. Ganz ohne Widerspruch blieb aber die Lehre vom blossen Kontakt auch auf dịesem Gebiete nicht. $\mathrm{Yie} \mathrm{mack}^{6}$ ) sah an vital gefärbten Präparaten nur einen Teil der Achsenzylinder frei auslaufen, während ein anderer an den Haarzellen durch Vermittlung einer becherförmigen, protoplasmatischen, mit dem Zellkörper verschmolzenen Zwischensubstanz endigt. Man hat es nach seiner Darstellung mit einem ganz eigentümlichen Verhälnis zu tun, das sich weder mit dem Begriff der Kontinuitä noch der Kontiguität deckt.

Krause ${ }^{7}$, der sich ebenfalls vorwiegend auf die Bilder der Golgischen und Ehrlichschen Methode stützte, stellte fest, dass die Achsenzylinder der Vestibularisfasern die Haarzellen an

1) $\mathrm{Ka}$ is er: Das Epithel der Crist. und Mac. ac. Archiv für Ohrenheillkunde, 1891, Bd. 32 .

$\Rightarrow$ v. L e n h o s ék: Die Nervenendigungen in der Mac. und Crist. ac. Anat. Hefte 1893.

3) van Ge h u chte n: Verhandlungen der anatomischen Gesellschaft in Wien, 1892.

4) Ramón y Caja1: Nuovo concepto de la Histologia de los centros nerv. Barcelona 1893.

5) Gastav Retzi s: Die Endigungsweise des Gehörnerven. Biologische Untersuchungen, N. F., Bd. II.

$\left.{ }^{8}\right) \mathrm{N}$ iemack: Maculae und Cristae acusticae. Merkel u. Bonnet. Anat. Hefte, I. Abt., 5. Heft, 1893.

7) Krause: Die Endigungsweise des N. acusticus im Gehörorgan. Anat. Anzeiger, Bd. XII, Ergänzungsheft. 
der Basis kelchförmig umfassen, ohne dass eine irgendwie deutliche Grenze zwischen nervöser und Zellsubstanz hervortrete. Es handle sich um eine ,ausserordentlich innige" Verbindung, die sich aber erst, wie embryologische Beobachtungen lebren, sekundär ausbilde.

Im schroffsten Widerspruch zur Kontaktlehre stehen die Ausfübrungen von Ayers. ${ }^{1}$ ) Dieser Autor behauptet, dass alle Fasern des N. VIII aus den Haarzellen ihren Ursprung nehmen, und dass demnach zwischen akustischen und olfactorischen Elementen kein Unterschied bestehe. „Die Haarzellen und die ihnen anhăngenden Ganglienzellen bilden eine morphologische Einheit." Ayers bediente sich gleichfalls der Golgischen Methode und führte seine so weit von der Majorität abweichenden Anschauungen auf ein besseres Gelingen seiner Imprägnationen zurück; die Kontaktvorstellungen der anderen Forscher sind nach seiner Meinung durch unvollkommene Schwärzungen der nervösen Elemente bedingt, welche dort, wo in Wirklichkeit noch gar keine Endigung sei, freie Ausläufer vortäuschen. Die Opposition gegen diese Thesen liess nicht lange auf sich warten, und Retzius selbst war es, der dem amerikanischen Autor Irrtümer in der Beschreibung und Deutung seiner Präparate zum Vorwurf machte.

Mit einer eigenen, sehr interessanten Methode hat dann $\mathrm{Held}^{2}$ ) die Sinnesorgane des Obrlabyrinthes untersucht. Er fand, dass die Haarzellen der Maculae resp. Cristae acusticae an ihrer Oberfläche vollständig von einem neurosomenreichen Neuritenprotoplasma bedeckt sind, welches aus der intraepithelialen Aufzweigung markloser Fäserchen des Nervus vestibularis entsteht. Die Bilder, welche dieser Forscher entwirft, gleichen vollkommen denjenigen, welche er schon früher von der Endausbreitung zentraler Neuriten an der Oberfläche der Ganglienzellen gegeben batte. Das Axoplasma zeigt eine netzartige Anordnung und ist mit dem Zellprotoplasma innig verwachsen.

Augenblicklich steht die Erforschung der peripherischen Nervenendigungen im Beginn einer neuen Periode, welche durch

1) Ayers: Über das periphere Verhalten des Gehörnerven etc. Anat. Anzeiger, Bd. VIII, 1893.

2) Hans Held: Zur Kenntnis des Cortischen Organes und der übrigen Sinnesapparate des Labyrinthes bei Säugetieren. Abh. der math.phys. Klasse der Kgl. sächs. Gesellschaft der Wissenschaften. Teubner, 1902. 
das Bekanntwerden der Silberreduktionsmethoden eingeleitet wird. Für das Gehörorgan sind dieselben bisher noch wenig benutzt worden.

An erster Stelle ist hier Ramón y $\mathrm{Cajal}^{1}$ ) zu nennen. Mit seinem der photographischen Technik nachgebildeten Verfithren untersuchte der berühmte spanische Histologe die Maculae und Cristae acusticae 17-19 tägiger Hühnerembryonen und fand dort zwei Arten von Nervenendigungen : erstens freie Ausläufer, welche den mit der Golgischen Methode von Retzius und Lenhossék dargestellten Endigungen gleichen, und zweitens kelchartige Gebilde, welche aus viel dickeren Achsenzylindern als die erstgenannten hervortreten und nur in der Gipfelpartie der Cristae anzutreffen sind. Diese nervösen Endkelche bilden einen zarten Fibrillenmantel um das untere Viertel oder Fünftel der Haarzellen, sind aber nach der Ansicht Cajals vom Zellkörper substantiell scharf getrennt.

A usserdem hat Ko $1 \mathrm{mer}^{2}$ ) mit derselben Nethode die Endigungsweise des Gehörnerven bei Rana esculenta untersucht. In einer Publikation aus dem Jahre 1904 gibt er an, dass die Endausläufer der Achsenzylinder teils mit schleifenartigen Figuren am unteren Pol der Sinneszellen endigen, teils als kleine Fibrillenbündel zum oberen Teil des Zellkörpers aufsteigen und ihn mit mehreren quer verlaufenden engen Windungen umfassen. Diese Darstellung liegt noch vollkommen im Rahmen der Kontaktlehre. Aber schon im nächsten Jahre erschien eine weitere Mitteilung ron $\mathrm{Kolm} \mathrm{er}^{3}$ ), in der er seinen Standpunkt wesentlich ändert, obgleich dasselbe Material zugrunde lag und dieselbe Färbemethode angewandt worden war. Jetzt behauptete der Autor, dass Neurofibrillen von der Seite her in die Sinneszellen eindringen und in ihrem In ner $n$ ein aus unregelmässigen Maschen zusammengesetztes Gitter bilden, das nur selten ganz vollständig imprägniert ist, aber meist deutlich in der Nachbarschaft des Kernes zur Ansicht

1) Ramón y Cajal: Association del método del nitrato da plata con el embrionario para el estudio de los focos motores y sensitivos. Trabajos del labor. de investigaciones biol. de la Univ. de Madrid. Tome III, 1904.

?) Kolmer: Über die Endigungsweise des Nervus octarus. Zentralblatt für Physiologie, Dez. 1904, Bd. 18, Nr. 20.

3) Kolmer: Über das Verhalten der Neurofibrillen an der Peripherie. Anat. Anzeiger 1905, pag. 560. 
gelangt. Das Kaliber dieser intrazellulären Fibrillen ist verhältnismässig dick. Die obersten Maschen des Gitters erreichen niemals die Zelloberfläche. Zwischen ibnen und der die Hörbaare tragenden Schlussplatte bleibe ein deutlicher Zwischenraum, in dem ein helles Korn zuweilen auffält. Neben diesen intrazellulären Endstrukturen der Fibrillen beschreibt er auch noch extrazellulär gelegene Endgebilde in Gestalt von eigentümlichen Schleifen und Ringen.

Als allgemeine Schlussfolgerung aus seinen Befunden geht hervor, dass die Neurofibrillen dort, wo man bisher Nervenendigungen annahm, nicht frei endigen, sondern entweder in Form einfacher Schleifen oder in Gestalt komplizierter Gitter, ohne Unterbrechung der Kontinuität, zu den Fibrillen der leitenden Bahn zurückkehren. In konsequenter Weise betont er dann auch, dass die Lehre von den freien Nervenendigungen und den primären und sekundären Sinneszellen revidiert werden müsse. Auch mit gewissen Annahmen der Neuronenlehre seien diese Resultate kaum vereinbar; denn die in n e rhalb einer Sinneszelle gelegenen Fibrillen könnten nutritiv nicht bloss von ihrer Ursprungsganglienzelle abhängig sein, sondern gewiss auch von der betreffenden Sinneszelle selbst, in der sie die Gitter bilden. Bemerkenswert ist, dass dieser Autor seine Ergebnisse als Zufallsprodukte bezeichnet, welche er dem $z$ ufalligen Gelingen einer neuen, wenig ausgebildeten Methodils verdankt.

Man sieht, dass die Angaben Kolmers bis zu einem gewissen Grade wieder mit der Darstellung übereinstimmen, welche die Forscher der Periode vor Golgi von den Sinnesepithelien und ihren Beziehungen zu den Nervenfasern entworfen baben. Die alten Kontroversen bestehen also weiter.

Unter diesen Umständen war der Versuch gerechtfertigt, mit einer neuen Fibrillenmethode die Nervenendstellen des Acusticus zu untersuchen. Wir bedienten uns der von Bielschowsky, angegebenen Nethode, welche auf der Aldehydreduktion ammoniakalischer Silberlösungen berubt und die besten Resultate an Gefrierschnitten liefert. Das Verfahren, welches ursprünglich für das zentrale Nervensystem angegeben ist, musste für die Darstellung peripherischer Nervenfasern einer Modifikation unterzogen werden, weil sonst die sich mitfärbenden Bindegewebselemente eine genaue Orientierung erschweren. Für diejenigen Histologen, 
welche unsere Angaben nachzuprüfen beabsichtigen, sei die Wethode kurz angegeben:

1. Die in Betracht kommenden Partien des Felsenbeines werden in $20 \%$ Formollösung fixiert und in $5 \%$ Salpetersäure entkalkt. Nach vollendeter Entkalkung werden sie entwässert und einige Tage in 20\% Formollösung zurückgebracht. Vor dem Schneiden empfiehlt es sich die Präparate etwa eine Stunde in Hiessendem Wasser abzuspülen, um das freie Formalin, welches den Gefrierprozess sehr erschwert resp. unmöglich macht, zu entfernen. Die so vorbehandelten Objekte lassen sich auf jedem Kohlensăuremikrotom leicht frieren und schneiden.

Das Schneiden uneingebetteten Materials wird zunächst jedem sonderbar erscheinen, der die zarten Epithel- und Nervenstrukturen des inneren Ohres kennt und weiss, wie leicht Verschiebungen und Zerreissungen in diesem Gewebe stattfinden. Tatsächlich aber ist die Gefriermethode bei einiger Übung dem Einbettungsverfahren mindestens gleichwertig, und häufig ist es uns gelungen, die topographischen Verhältnisse so intakt zur Darstellung zu bringen, wie man es nur wünschen mag. Ein grosser Vorzug dieses Verfahrens vor den Einbettungsmethoden besteht darin, dass vor der Färbung kein Alkohol mit dem Gewebe in Berührung kommt; erstens werden dadurch Schrumpfungen vermieden und zweitens bleibt die Färbbarkeit der Neurofibrillen eine bedeutend bessere.

2. Die Schnitte werden vom Messer in destilliertes Wasser übertragen und kommen dann für 24 Stunden bei Zimmertemperatur in eine $4 \%$ Lösung von Argentum nitricum (Aufbewahren im Dunkeln!).

3. Die Schnitte werden nach kurzem Durchziehen durch destilliertes Wasser in eine Silberoxydammoniaklösung übertragen, welche in folgender Weise hergestellt wird: $\mathrm{Zu} 5 \mathrm{ccm}$ einer $20 \%$ Arg. nitric.-Lösung fügt man (am besten in einem Messzylinder) fünf Tropfen einer $40 \%$ Natronlaugenlösung. Der entstehende dunkle Niederschlag wird durch tropfenweisen Zusatz von Ammoniak aufgelöst, bis die Flüssigkeit vollkommen klar geworden ist oder nur noch einen leicht gelblichen Schimmer aufweist. Dann giesst man $20 \mathrm{ccm}$ destilliertes Wasser hinzu. Ton Wichtigkeit ist, dass kein zu starker Ammoniaküberschuss, der am Geruch ohne weiteres erkennbar ist, in der Lösung besteht. 
In dieser Flüssigkeit bleiben die Schnitte einige Minuten, bis sie einen bräunlichen Ton angenommen haben. (Glasschälchen!)

4. Dann kommen die in schwach angesäuertes Wasser. Es genügt ein Tropfen Eisessig für $20 \mathrm{ccm}$ Aqua dest. Hier weicht der braune Ton nach kurzer Zeit einer etwas helleren Nuance, und jetzt erfolgt die Übertragung in

\section{5. $20 \%$ Formalinlösung.}

Die Reduktion vollzieht sich ziemlich langsam. Man lässt die Schnitte so lange in dieser Lösung, als noch weisse Wölkchen aus ibnen aufsteigen. Damit ist die eigentliche Färbung vollendet.

Bei älteren Objekten, welche längere Zeit in der Konservierungsflüssigkeit gelagert hatten, erzielt man gute Resultate nur dann, wenn man die Prozeduren 3-5 Mal wiederholt, wobei zu beachten ist, dass man formalinhaltige Schnitte nicht unmittelbar in ammoniakalisches Silberoxyd bringen darf, sondern dieselben vorher längere Zeit wässern muss. Diese Verdoppelung der Prozeduren ist übrigens nie von Nachteil und erhöht unter allen Umständen die Sicherheit des Gelingens der Färbung. Um recht brillante und unvergängliche Präparate $z u$ erhalten, empfiehlt es sich, eine Vergoldung und Fixierung mit Natrium thiosulfat in der bekannten Weise folgen zu lassen. ${ }^{1}$ ) Das Entwässern und Aufhellen der Präparate geschieht wie gewöhnlich in Alkobol von steigender Konzentration und $5 \%$ Carbolxylol. Einschliessen in Canadabalsam.

Wie aus den beigegebenen Abbildungen ohne weiteres hervorgeht, besteht der Hauptvorzug dieses Verfahrens vor der Methode Ramón y Cajals darin, dass es die nervösen Elemente häufig mit quantitativer Vollständigkeit auf der ganzen Fläche des Schnittes zu Gesichte bringt, und nicht in einzelnen tupfenförmigen Gebieten. Man überblickt deswegen nicht selten in einem einzigen Präparate die topographischen Verhälnisse vollkommen und kann auf jede subjektive Kombination verzichten.

Ein zweiter Vorzug besteht darin, dass man nicht nur die Kerne der Gewebszellen, sondern auch deren Grenzen scharf and deutlich sieht, so dass Zweifel über die genaue Lage der Neurofibrillen zur Sinneszelle, über intra- oder extrazelluläre Strukturen, kaum auftauchen können. Schliesslich werden aber auch noch

1) Bielschowsky: Silberimprägnation der Neurofibrillen. Journ. f. Neurol. u. Psychologie u. Neurol. Zentralblatt, 1903, Bd. 22, pag. 999. 
protoplasmatische Strukturen in den Zellkörpern sichtbar, welche der Cajalsche Reduktionsprozess nicht wahrnehmbar macht.

Zum Vergleich und zur Kontrolle haben wir das von Bielschowsky angegebene Blockverfahren und die verschiedenen von $\mathrm{Cajal}$ angegebenen Reduktionsmethoden herangezogen, ohne dass uns jedoch die Erfolge besonders befriedigt haben. Als Untersuchungsmaterial bevorzugten wir das Gehörorgan von Meerschweinchen, weil bei diesen Tieren die Schnecke nur von einer dünnen Knochenwand umhüllt in die Paukenhöhle hineinragt. Dieses Verhalten ermöglicht bekanntlich eine rasche Entkalkung und ausserdem eine sichere Orientierung der Schnittebenen auf dem Mikrotom. Für einzelne entwicklungsgeschichtliche Fragen konnten wir mehrere gut gelungene Serien von menschlichen Embryonen und solche von Scyllium can. und Salamandra mac., die nach dem Bielschowskyschen Blockverfahren imprägniert waren, heranziehen.

Wenn wir jetzt zur Beschreibung unserer Präparate übergehen, so sei bemerkt, dass dieselben nicht nur an den Nervenendstellen klare Bilder lieferten, sondern die ganze Nervenbalın von den Ursprungsganglien an mit grosser Deutlichkeit zu Gesicht brachten.

\section{Ganglion vestibulare und radix vestibularis.}

Die Zellen des S carpa schen Ganglions (Vestibularganglion) zeigen im Fibrillenbilde die gleiche abgerundete Form wie im Golgi- und Nissl präparate. Die Fibrillen des Zellkörpers bilden ein grobes Gitterwerk von ähnlichem Gefüge, wie es an den Spinalganglienzellen öfters beschrieben worden ist. Es erinnert an den Querschnitt eines porösen Schwammes; nur sind hier die Bälkchen des Schwammgefüges im allgemeinen etwas zarter und die. Maschen etwas enger als dort. Die beiden Fortsätze entspringen, wie Fig. 3 auf Tafel V zeigt, von entgegengesetzten Polen des Zellkörpers, und ihre Fibrillen lassen sich kontinuierlich in dessen Gitterwerk verfolgen. Beachtenswert ist dabei, dass das Kaliber der beiden Fortsätze sich in gesetzmässiger Weise unterscheidet. Während der peripherische, zum Sinnesepithel hinziehende, nur ein zartes homogenes Fädchen bildet, besteht der zentrale aus einem starken Fibrillenbündel, welches auf der Anfangsstrecke bis zum Ort der 
Markumkleidung eine grössere Menge plasmatischer Substanz in sich schliesst. Der zentrale Fortsatz erinnert also an die Struktur der Dendrit en zentraler Nervenzellen. Diese Differenz im Kaliber der Fortsätze kommt auch im Golgibilde klar zum Ausdruck und steht ganz im Gegensatz zu dem, was man an den Fortsätzen der Spinalganglienzellen beobachtet hat. Bereits von Cajal ist bemerkt worden, dass die beiden Fortsätze der Vestibularganglienzellen in ihrem Kaliber voneinander abweichen; nur hat er den peripherischen Fortsatz als den stärkeren, den zentralen als den zarteren bezeichnet. Diese Ansicht ist offenbar durch eine besondere Wertschätzung des Gesetzes der "dynamischen Polarisation" bedingt worden, nach welchem die nach der Peripherie ziehenden, zentripetal leitenden Fortsätze dieser Ganglienzellen als umgewandelte Dendriten angesehen werden mussten. Schon v. Lenhossék ${ }^{1}$ ) hat dies bemerkt und richtig gestellt. Nach seiner Meinung hängt das stärkere Kaliber der einen Faser mit der grösseren Länge der Strecke zusammen, die sie zurückzulegen hat, und in der Tat wird man in den geschilderten Befunden nichts als einen neuen Beleg für die bekannte Tatsache $z u$ suchen haben, dass ein proportionales Verhăltnis zwischen Lünge und Durchmesser des Achsenzylinders bestebt. (Allerdings ist auch diese Regel nicht ohne Ausnahme.) Die Ursprungsstellen der Fortsätze sind übrigens nicht bei allen Zellexemplaren rein oppositipole. Man sieht fast in jedem Schnitt einige, bei denen dieselben einander genahert sind (cf. Fig. 3, Taf. V). Es handelt sich offenbar um Übergangsformen zu dem gewöhnlichen Typus der Spinalganglienzelle. Noch einen Schritt weiter, und es verschmelzen die beiden Fortsătze zu ein em Stammfortsatz, wie er jenen Zellen eigentümlich ist. Multipolare Ganglienzellen sind von Ayers und Cannieux im Ganglion vestibulare beschrieben worden Derartige Gebilde haben wir nie gefunden, ebensowenig Zellformen, welche an die sympathischen Zellelemente Dogiels erinnert hätten.

Die peripherischen Fortsätze verlassen das Ganglion als derbe Markfasern und sind durch das subepitheliale, lockere

1) v. Lenhossék: Die Nervenendigungen in Maculae und Cristae acusticae. Beitr. zur Histologie des Nervensystems und der Sinnesergane. Wiesbaden. Bergmann 1894 und l. c. 
Bindegewebe der Maculae und Cristae hindurch bei erwachsenen Tieren als fibrillär gestreifte Bänder zu verfolgen. Eine derartig deutlich auf den markhaltigen Strecken der Fasern hervortretende fibrilläre Streifung ist sonst in Reduktionspräparaten eine Seltenheit; sie spricht dafür, dass der Narkmantel trotz des starken Kalibers der Faser nur ein dünner ist und dass das Myeloaxostroma, d. i. die tingierbare Acbsenzylindersubstanz. welche auch im Silberbilde die homogene Schwärzung markhalt ig e r Nervenfasern bedingt, nur in geringer Menge vorhanden ist. Beim Durchtritt durch die Basalmembran verlieren die Fasern ihre Markscheiden und ziehen als derbe Fibrillenbündel, in denen jedes einzelne Füdchen noch schärfer als vorher zu erkennen ist, weiter. Dabei ändern viele ihre Verlaufsrichtung, indem sie sich gabelförmig teilen und in spitzen Winkeln auseinanderweichen. Ohne vorhergehende Teilung und ohne jede Abweichung aus der ursprünglichen Richtung ziehen nur die zu den Kulminationsgebieten der Cristae gehörigen Fasern weiter, welche sich auch im Fibrillenbilde, wie Ramón $\mathrm{y} C \mathrm{C}$ jal an Hühnchenembryonen festgestellt hat, durch ein besonders starkes Kaliber auszeichnen. In den Maculae und den seitlichen Abhüngen der Cristae ziehen die Teiläste durch die Schicht der tiefen Fadenzellen schräg nach aufwärts, bis sie in das Niveau der unteren Haarzellenenden gelangen. Hier lassen sich weitere Verästelungen in grosser Zahl beobachten, deren Verlauf der Obertlache des Epithels parallel gerichtet ist; es kommt auf diese Weise zu den bekannten Plexusformationen, welche sich als eine besondere, scheinbar gut begrenzte Schicht schon bei schwacher Vergrösserung bemerkbar machen. Echte Anastomosen zwischen benachbarten Fasern sind hier keine Seltenheit. In Übereinstimmung mit den Angaben früherer Autoren (Niemack) hat Kolmer sie auch in Cajalschen Reduktionspräparaten gefunden. Wir sahen sie am deutlichsten in Horizontalschnitten, welche der Oberfläche der Maculae parallel gerichtet waren.

Bevor wir das Schicksal der nervösen Elemente weiter verfolgen, müssen wir einen Blick auf die Zellen der Epithelschicht werfen, welche in unseren Präparaten ein eigenartiges Aussehen hat (Fig. 4-7,Taf.V). Am weitesten nach aussen ist dieselbe gegen das Lumen des häutigen Labyrinthes hin durch eine ziemlich breite, 
homogene Membran begrenzt, welche schon Pritchard ${ }^{1}$ ) und $\mathrm{Kaise} \mathrm{r}^{2}$ ) genau beschrieben haben. $\mathrm{Ka}$ is er hat sie als eine wahre "Limitans" bezeichnet, welche von den Zellen unabhăngig sein soll. Von dieser Limitans gehen auf senkrechten Querschnitten in unseren Präparaten Fortsätze nach unten, tief in die Schicht der Fadenzellen hinein. Sie haben die Gestalt gleichschenkliger Dreiecke, deren Basis der Limitans aufsitzt, und deren Spitzen bis unter die Mitte der Haarzellkörper, 'bis in das Niveau der Fadenzellenkerne reichen. In der Regel ist die Substanz dieser Fortsätze etwas dunkler gefärbt und nicht so homogen wie die der Aussenmembran selbst. An dünnen Schnitten lässt sich nicht selten eine Art Wabenstruktur an ihnen feststellen. Manchmal findet man dort auch starre farblose Fasern, welche sich bei Anwendung starker Blenden als glänzende Einschlüsse der Fadenzellen erkennen lassen. Das sind offenbar Stützstrukturen, die histiochemisch den Cuticularsubstanzen verwandt zu sein scheinen. Held hat sie als erster genau beschrieben und abgebildet. Er kennzeichnet sie als intrazelluläre Stützfasern der Schultzeschen Fadenzellen, die nach unten teilweise bis zur Basalmembran reichen und oben pinselartig an einem im Bereich der Haarzellen besonders ausgebildeten, verdickten Schlussleistennetz angeheftet sind. Sie sind den Stützfasern in den Deitersschen Zellen und in den Pfeilern des Cortischen Organs gleichwertig. Dadurch, dass diese dreieckigen Gebilde die Ränder der Haarzellen nicht berühren, sondern durch eine ziemlich breite Lücke von ihnen getrennt bleiben, erhält das Gesamtbild der Epithelschicht ein arliadenartiges Aussehen. Es reiht sich ein Spitzbogen an den anderen, und in diesen sind die Haarzellen wie Glocken aufgehängt. Die Flimmerhaare sind bei den von uns untersuchten Tieren stets zu gröberen Borsten verklebt gewesen. Die Ansatzstellen der Borsten korrespondierten immer mit den Insertionsstellen der darunter liegenden Haarzellenköpfe. Eine Längsstreifung war in den Borsten nicht zu konstatieren, ebensowenig wie ein substantieller Zusammenhang mit den genannten Zellen.

1) Pritchard: Quart. Journ. of microscop. Science, Vol. 16, new Ser. 1876, pag. 398 .

2) Kaiser: Das Epithel der Maculae und Cristae. Arch. f. Ohrenheilkunde, Bd. 32, pag. 182, 1891. 
Die Fadenzellen selbst zeigen sich als rundliche Gebilde mit dunklem homogenem Protoplasmaleib und einem zentralen Kern, in welchem wieder ein oder zwei Kernkörperchen auffallen. Die fadenförmigen Fortsätze treten immer dentlich hervor, enthalten zuweilen jene hellen Stützfasern und sind häufig über weite Strecken zu verfolgen. So sieht man, dass in der Regel ein ganzes Bündel von Fortsätzen sich in die Spitze der geschilderten Dreiecke hineinsenkt, und dort $\mathrm{zu}$ einer homogenen Masse verschmilzt, ein Befund, welcher darauf hinweist, dass jene Gebilde als eine von den Fadenzellen produzierte eigenartige Interzellularsubstanz aufzufassen sind. Die Hohlräume, in welchen die Haarzellen liegen, werden basalwärts und in den unteren Partien ihrer seitlichen Grenze von den Fadenzellenkörpern gebildet. Unverkennbar sind hier gewisse örtliche Beziehungen dieser Zellen zu den Fibrillenbündeln der Nervenfasern, denen sie sich in ähnlicher Weise anzuschmiegen scheinen, wie das die Schwannschen Zellen auf den marklosen Endstrecken sensibler Hautnervenfasern tun.

Bemerkenswert ist ferner die Struktur der Haarzellen selbst. In den bekannten flaschen- oder keulenförmigen Zellkörpern sieht man einen grossen farblosen Kern, in dem sich häufig zwei dunkle Kernkörperchen abheben. Im Zelleib lassen sich drei Zonen unterscheiden, welche etwa dem Hals, der Seitenwand und dem Boden der Flasche entsprechen. Der Hals zeigt bei Anwendung stärkster Vergrösserung eine deutliche Längsstreifung, welche sich fast immer in eine grosse Zahl zartester Körnchen autlösen lässt. Manchmal hat man den Eindruck, als ob diese Körnchenstrukturen der mikroskopische Ausdruck für das Vorhandensein längsgerichteter Waben sind, deren Querwănde sich als kleinste Mikrosomen besonders abheben. Während die der Seitenwand der Flasche entsprechende Partie des Zellkörpers einen homogenen schmalen Streifen bildet, findet man im Bodenteil wieder eigenartige Körperchen. Diese haben auch hier das Aussehen runder Körnchen oder Kügelchen, weisen aber ein bedeutend stärkeres Kaliber auf, als die erwähnten Gebilde im Halse. Häufig kann man sie bis in den basalen Bezirk der Zelle und zwar bis zur aussersten Randzone verfolgen, wo die Verbindung mit den Neurofibrillen erfolgt. Das basale Zellgebiet enthält schliesslich noch besonders bemerkenswerte Einschlüsse von eigentümlich ringförmiger Gestalt, welche wegen ihres 
morphologischen Zusammenhanges mit den Neurofibrillen als nervöse Gebilde anzusprechen sind. Von ihnen wird später die Rede sein.

Verfolgen wir jetzt den Verlauf der nervösen Fasern weiter, so sehen wir, dass von den ziemlich groben Ästen, welche die plexiforme Schicht unter dem Niveau der Haarzellen bilden, zarte $\ddot{A}$ stchen aufsteigen, welche in zwei verschiedene Arten von Endgebilden auslaufen.

Ein kleiner Teil von ihnen geht in die Zwischensubstanz zur Spitze der dreieckigen Fortsätze, welche von der äusseren Grenzmembran in die Schicht der Haarzellen vordringen (Fig. 6, Taf. V). Hier erfolgt meist eine dichotomische Teilung, und unmittelbar darauf eine Auflösung des Fibrillenbündels zu langen Knäueln, in denen die Einzelfibrillen in einfachen Schlingentouren in sich zurückkehren. Diese Endknäule enthalten ausser den Fibrillen immer noch eine Masse plasmatischer Substanz, welche gleichmässig zwischen den Fädchen verteilt ist. Es scheint, dass auch die spezifische Zwischensubstanz der Epithelschicht zwischen die Fibrillen eindringt; denn es ist mitunter sehr schwer, die zarten fibrillären Elemente von den Längswänden jener eigentümlichen Wabenstrukturen zu sondern, welche die Substanz jener Dreiecke bilden. In jedem Falle tritt hier eine ausserordentlich innige Verschmelzung aller in Betracht kommenden Gewebskomponenten ein. Stellt man sich vor, dass diese Endschlingen von den kompakten Niederschlägen des Chromsilbers zu Silhouetten umgewandelt werden, so wird man kaum daran zweifeln können, dass wir hier jenefreien Endausläufer vor uns laben, welche zuerst den Golgiforschern am klarsten vor Augen getreten sind (Retzius, Lenhossélk). Im Golgibilde liegen die keulenförmïgen und rundlichen Endvarikositäten, welche unseren Fibrillenknäueln entsprechen, der Obertläche der Sinneszellen dicht an, während sie in unseren Präparaten durch eine mehr oder weniger breite Lücke von ihnen getrennt sind; auch reichen sie dort meist nicht so weit an die aussere Oberfläche des Organs heran wie hier. Diese Differenzen sind durch die Verschiedenartigkeit des Untersuchungsmaterials bedingt. Die Chromsilberimprägnation liefert ihre besten Resultate an Embryonen, welche daher fast ausschliesslich zur Betrachtung herangezogen wurden. Die embryonalen Organe unterscheiden sich aber bekanntlich nicht unwesentlich von den fertig ausgebildeten. Es fehlt ihnen vor 
allen Dingen jene eigentümliche Zwischensubstanz, welche später die Haarzellen voneinander trennt, und deshalb liegen diese Zellen und die zwischen ihnen endigenden Nervenfasern viel näher beieinander, als es bei unseren reifen Objekten der Fall ist. Dazu kommt, dass sich mit zunehmender Ausbildung der äusseren Limitans und ihrer dreiseitigen Fortsätze ein Saftlückensystem entwickelt, welches alle Haarzellen umgibt und wahrscheinlich mit den Lymphspalten des subepithelialen Bindegewebes kommuniziert ( $\mathrm{K}$ a iser). Beide Momente können eine Verschiebung der freien Ausläufer von der Oberfläche der Zelle in die Substanz des Zwischengewebes zur Folge haben. Es sei an dieser Stelle erwähnt, dass basale, zur tiefen Schicht der Stützzellen hinstrebende Ausläufer, wie sie v. Lenhossék in seinen Grolgipräparaten gefunden hat, im Fibrillenpräparat nicht von uns gesehen wurden.

Der weitaus betrăchtlichere Teil der Endfasern tritt an die basale Partie der Haarzellen selbst heran; dort weichen die Fibrillen auseinander und umfassen den unteren Teil der Haarzellen in der Weise, wie etwa eine Vogelkralle eine Kugel umspannt. Je nach dem Grade der Imprägnation ist in quantitativer Hinsicht das Bild, welches diese perizellulälen Endverzweigungen bieten, ein etwas verschiedenes. Da, wo nur wenige Fibrillen gefärbt erscheinen, umfassen sie den Rand der Zelle und sind etwa nur bis zur Höbe des oberen Kernrandes zu verfolgen (Fig. 6, Taf. V). Ausserdem finden sich Bilder, in denen del ganze Zelleib von feinsten Endfäserchen eingehüllt ist, die wieder durch zarte Anastomosen miteinander verbunden erscheinen (Fig. 5, Taf. V); und schliesslich findet man gar nicht selten Zellenexemplare, welche von der Basis bis zur Zellspitze umsponnen sind von einem engmaschigen Geflecht gröberer und feinerer Fibrillen (Fig. 7, Taf. V). Dass es sich hier nicht um prinzipiell verschiedene Endigungsarten, sondern nur um graduell verschiedene Darstellungen identischer Formationen handelt, liegt auf der Hand. Aber nicht nur die Fibrillen des Achsenzylinders, sondern auch die plasmatische Substanz nimmt an der Umhüllung der Sinneszellen teil; man sieht das deutlich an gut vergoldeten Präparaten. Das interfibrilläre Axoplasma nimmt nämlich kurz vor den Endstellen nicht unerbeblich an Masse zu und hebt sich durch seinen rötlichen Ton deutlich von den Fibrillen 
ab. Diese plasmatische Substanz begleitet die Fibrillen bis in die Endnetze und füllt gewissermassen die Maschen in denselben aus.

Von besonderer Bedeutung aber ist in allen Präparaten, bei denen diese perizellulären Endstrukturen auch nur einigermassen deutlich hervortreten, folgender Befund. Man sieht im basalen Teil der Haarzellen scharf konturierte ring förmige Gebilde, welche sich ganz in demselben scbwarzen Farbenton präsentieren, wie die Nervenfädchen an ibrer Oberfäche. Manchmal sind diese Ringe über die Fläche gebogen und nähern sich dann der Pessarform; manchmal zeigen sie wellige Linien; immer aber handelt es sich um echte Ring for mati o n e n, bei denen das gefärbte Fädchen in sich selbst zurückläuft, - und nicht etwa um enge Spiralen, deren Windungen durch eine zu starke Imprăgnation miteinander verklebt worden sind. Am beweiskräftigsten sind in dieser Hinsicht gerade diejenigen Präparate, bei denen die Färbung sonst nicht besonders gelungen ist. In solchen Objekten beben sie sich nämlich mit dem denkbar schärfsten Kontrast als in sich geschlossene, vollkommene Ringe von der Nachbarschaft ab (Fig. 6a, Taf. V). (Bemerkenswert ist, dass gerade diese bisher nicht beschriebenen Gebilde sich mit der Silberaldebydmethode ausserordentlich leicht darstellen lassen ) An derartigen Präparaten erkennt man auch, dass die Lage der Ringe eine intrazelluläre ist. Man findet nämlich gar nicht selten Stellen, in denen der untere Teil des Kernes in das Lumen des Ringes hineinragt. Weniger häufig drängen sich diese Ringe zwischen Kern und Seitenwand der Zelle.

Es kann nun die Frage aufgeworfen werden, ob wir es hier mit einer nervösen Endstruktur oder mit einem eigentümlichen Differenzierungsprodukt der Sinneszelle selbst zu tun haben? Das mikrochemische Verhalten der Ringsubstanz und die Tatsache, dass man zuweilen fibrilläre Verbindungsbrücken $z$ wischen den perizellulären Fädchen und den Ringen selbst findet, sprechen entschieden für die erste Auffassung. Es kann unseres Erachtens also keinem Zweifel unterliegen, dass wir es hier mit einem ganz eigenartigen Endgebilde im Innern der Sinneszellen zu tun haben.

Bielschowsky hat mit seiner Methode die Nervenendstellen der Haut und der höheren Sinnesorgane bearbeitet, ohne 
jemals einer ähnlichen Ringbildung begegnet zu sein, während er dichte perizelluläre Netzformationen auch an anderen Stellen getroffen hat (z. B. an Sinneszellen in der Nasenschleimhaut von Centetes und an grossen bläschenförmigen Sinneszellen in der Haut der Hirudineen).

Die häufig wechselnde Lage, die das Gebilde am Boden der Zellflasche einnimmat, kann vielleicht die Vermutung wachrufen, dass es ein gewisses $\mathrm{Ma} \beta$ von $\mathrm{Bew}$ e glichkeit in dem weichen Protoplasma der Umgebung hat. Die funktionelle Bedeutung des Ringgebildes ist natürlich aus der mikroskopischen Betrachtung allein nicht zu erschliessen. Seine Lage macht es aber wahrscheinlich, dass es sich um einen Übertragungsmechanismus handelt, der die Bewegungen des Protoplasmas der Zelle gleichmässig auf die an ihrer Aussenfläche gelegenen Fibrillen weitergibt und so eine vollkommene Ausnutzung der auf die Zelle wirkenden Reize bei der Umschaltung ermöglicht.

Die perizellulären Fibrillennetze haben im Golgibilde ihr offensichtliches Analogon in jenen Becher- und Kelchstrukturen, welche von $\mathrm{Ka}$ iser, $\mathrm{Niemack}$ und besonders eingehend von R. Krause beschrieben worden sind. Die Fibrillenpräparate bestätigen auch die Anschauungen, welche sich $\mathrm{Kr}$ a use gebildet hat; denn es besteht in unseren Präparaten, auch wenn man von dem intrazellulären Ring und seinen Verbindungsfädchen mit dem Aussennetze vollkommen absieht, eine ausserordentlich innige Verschmelzung von Nerv und Sinneszelle. Und diese Verbindung ist um so vollständiger, als nicht nur die Fibrillen, sondern auch die plasmatis che Substanz der Achsenzylinder an ihr teilnehmen. Eine Grenze zwischen Axoplasma und Zellplasma ist an unseren vergoldeten Silberpräparaten überhaupt nicht $z u$ erkennen.

Auch bei der vitalen Methylenblaufărbung, welche im wesentlichen eine Axoplasmafärbung ist, kommt die Verschmelzung beider Substanzen dadurch zum Ausdruck, dass eine dunkel tingierte Randzone allmählich in das hellere zentrale Zellplasma übergeht. Von einem blossen Kontakt kann unseres Erachtens demnach $\mathrm{nicht}$ die Rede sein. Man wird zum mindesten von einer Concrescenz reden müssen, die um so vollständiger ist, als die Endausläufer der Nervenfasern bis in das Innere der Zelle einuringen und dort die beschriebenen Ringformationen bilden. 
Kolmer hat, wie wir oben bereits erwähnt haben, intrazelluläre Fibrillenstrukturen nervöser Art beschrieben, die ein aus unregelmässigen Maschen zusammengesetztes Gitter bilden und in kontinuierlichem Zusammenhange mit Nervenfäserchen stehell, welche von der Seite her in die Sinneszellen eindringen. Wir haben in unseren Präparaten analoge Bildungen nicht gesehen, und halten es für möglich, dass dieselben, wenn es sich hier wirklich um intrazelluläre Strukturen handeln sollte, durch protoplasmatische Waben, deren Wände unter dem Einfluss warmer Silberlösungen verbacken waren, vorgetäuscht wurden. Die von Kolmer beschriebenen extrazellulären Ring- oder Pessarformationen sind vielleicht mit unseren Ringen identisch und möglicherweise nur wegen einer unscharfen Darstellung der Zellgrenzen, die in Cajalschen Präparaten vorkommt, ungenau lokalisiert worden.

Mit Bezug auf die schon oben erwähnte Arbeit von Cajal wäre noch nachzutragen, dass die perizellulären Endformationen, welche er an seinen Hühnchenembryonen nur auf dem Gipfel der Cristae gefunden hat, an unserem Material überall vorhanden waren, in den Maculae ebenso zahlreich wie in den Cristae. Die Beschränkung seiner Becher auf die basale Partie der Zellenoberfläche erscheint bedingt durch das frühe Entwicklungsstadium seiner Objekte.

Der Nachweis der intrazellulären Fibrillenringe in den Haarzellen musste uns die Frage nahelegen, ob jene Zellen wirklich nur sekundäre Sinneszellen sind, oder ob sie nicht wenigstens einem Teil der Vestibularisfasern ihren Ursprung geben. Der Zufall brachte uns ein Material in die Hände, welches für die Beantwortung dieses Problems nicht ungeeignet war, nämlich zwei menschliche Embryonen aus dem Anfang und dem Ende des zweiten Schwangerschaftsmonates von 10 resp. $15 \mathrm{~mm}$ Steissnackenlange. ${ }^{1}$ ) Sie wurden nach Bielschowsky en bloc imprägniert, in Paraffin eingebettet und in lückenlose Horizontalschnitte von durchschnittlich $5 \mu$ Dicke zerlegt.

Fig. 8, Taf. V zeigt das Hörbläschen des $10 \mathrm{~mm}$ langen Fmbryos im Übersichtsbilde bei mittelstarker Vergrösserung. Die Wand desselben wird gebildet von einem mehrschichtigen Epithel-

1) Die Überlassung dieses Materials verdanken wir dem Frauenarzt Herrn Dr. Max Goldschmidt. 
belag, dessen Zellen auf der lateralen, in der Abbildung nach oben gerichteten Seite etwas grösser erscheinen als auf der medialen. Jedoch ist die mediale Wand dicker und zellreicher als die laterale. An der hinteren Circumferenz des Bläschens liegt ein mächtiger Haufen von rundlichen Zellen, welcher der Anlage des Ganglion acusticum entspricht. Eine örtliche Trennung zwischen vestibularem und cochlearem Anteil ist noch nicht vorhanden; aber man sieht bereits, dass ein lateraler, durch stark kalibrige Nervenfasern gekennzeichneter Abschnitt sich von einem medialen scheidet, der zahlreiche feinkalibrige Nervenfädchen führt. Die Zellen des Ganglion zeigen sich als rundliche Gebilde mit einem relativ grossen Kern und einem schwachen Protoplasmasaum, welcher bei vielen auf einer Seite des Kernes einen ausserordentlich feinen Fibrillenknäuel enthält. Bei einer grossen Zahl dieser primitiven Ganglienzellen lassen sich Fortsattze erkennen, welche zum Teil nach dem Sinnesorgan, zum Teil nach dem Gehirn gerichtet sind. Auch bipolare Elemente sind bereits vorhanden. In dem zentralen Gebiete des Ganglions liegen aber noch zablreiche Zellen, welche keine fibrilläre Differenzierung ihres Protoplasmasaumes aufweisen. Dieses faserarme, zentrale Gebiet liegt einem nach hinten gerichteten, stumpfen Fortsatz des Hörbläschens (bei a) dicht an. Man wird nicht fehlgehen, wenn man die laterale Partie als die Anlage des Scarpaschen Ganglions bezeichnet und die mediale mit dem spiiteren Ganglion spirale identifiziert. Für die Beantwortung unserer Frage ist es nun von Interesse, die aus dem Ganglion heraustretenden Fasern weiter bis in die Epithelwand hinein zu verfolgen. Das gelingt bei dem scharfen Kontrast, mit dem sich die schwarzen Nervenfasern von der Umgebung abheben, ganz leicht. Man kann einen Vestibularstrang aus dem lateralen Bezirk des Ganglions weit an der Circumferenz der entsprechenden Wand des Bläschens bis zu dessen vorderem Pol verfolgen und feststellen, dass einzelne Fäserchen aus diesem Strange rechtwinklig in das Epithel abbiegen und sich zwischen den Zellen verzweigen. Irgend ein Z usammenhang zwischen Fasern und Epithelien ist in diesem Stadium noch $\mathrm{nicht}$ zu erkennen. Das Eindringen der Neurofibrillen in die Hörbläschenwandung ist in Fig. 9, Taf. V wiedergegeben, welche der Stelle bei a in Fig. 8 entspricht. Nur ist sie hier bei stärkerer Vergrösserung gezeichnet. Ganz dasselbe 
gilt von dem aus dem medialen Rande des Ganglienhaufens hervorgehenden Cochlearnerven.

In einer anderen Hinsicht ist ferner das Präparat noch sehr bemerkenswert. Es zeigt nämlich, dass in einem bestimmten Gebiete der Hörbläschenwandung eine scharfe Scheidung von Epithelien und dendahinter liegenden Zellen des Ganglions nicht durchführbar ist. Man sieht vielmehr, dass in der Berührungszone zahlreiche Kerne mit den Nervenfasern in den Bereich der Wandung vordringen und sich dort mit den Epithelien zu vermischen scheinen. Man kann beide Zellarten an der verschiedenen Gestalt und Färbung ihrer Kerne noch einigermaßen unterscheiden. Die Kerne der Epithelien haben die Form breiter, ziemlich dunkler Stäbchen, wăhrend die Kerne der aus dem Ganglion vordringenden Zellen das Aussehen hellerer, runder Bläschen besitzen. Untersucht man das topographische Verhalten der Nervenfüserchen zu jenen Kernen bei stärkster Vergrösserung, so hat man gar nicht selten den Eindruck, dass sich Kernketten an den Fibrillenbündeln entlang ziehen und dass sie mit zarten Wellenlinien sich dem Rande der Zellen mehr oder weniger genau anschmiegen. Ob diese Zellen für das Lüngenwachstum der Fasern von einschneidender Bedeutung sind, ob sie als Fibrillenbildner oder Nervenzellen im Sinne Apathys anzusprechen sind, das ist eine Frage, die wir hier nicht erörtern wollen, und für welche unsere Präparate auch kein ausreichendes Substrat liefern. Sicher aber ist, dass in einem früheren Stadium der Entwicklung aus der Ganglienanlage Zellen gemeinschaftlich mit den Nervenfasern in die Hörbläschenwandung eindringen und sich vorwiegend in den Aussenschichten derselben verbreiten. Später sind sie dann von den Stützzellen nicht mehr zu unterscheiden. Dieser Befund berechtigt uns zu der Vermutung, dass ein Teil der späteren Fadenzellen möglicherweise von den Zellen der Ganglienanlage abzuleiten ist. Es liegt nahe, diese einwandernden Zellen mit den $\mathrm{S} c \mathrm{~h}$ w a n n schen Zellen zu identifizieren, welche nach den Untersuchungen $\mathrm{Har}$ r i sons als Abkömmlinge der Ganglienleiste aufzufassen sind. -

In dem zweiten uns zur Verfügung stehenden Embryo hatten wir topographisch schon weit übersichtlichere Verhältnisse vor uns. Hier hatten sich bereits deutliche Endorgane aus dem Zellgefüge der Wandung differenziert. Fig. 10, Taf. V zeigt uns an 
einem Horizontalschnitt zwei Vestibularisendstellen in den Cristae der Ampullen von zwei sich hier ausstülpenden Bogengängen. $\mathrm{Zu}$ diesen als flache Hügel hervortretenden Bezirken sieht man zwei starke Bündel zarter Nervenfäserchen hinziehen, welche bis zu ihrem Ursprungsganglion, vor dem sie sich zu einem starken Stamm vereinigen, verfolgt werden können. Der Gehalt an nervösen Fasern im Ganglion selbst ist grösser als in dem früheren Stadium; bipolare Zellen mit gegenständigen Fortsätzen sind häufig vorhanden. Es kommen aber auch zahlreiche fortsatzlose Zellen vor, welche sich noch eine beträchtliche Strecke in den Nervenstamm hinein verfolgen lassen. Nur unmittelbar vor der Wand des Gehörbläschens verschwinden sie fast vollkommen. In den Endhïgeln selbst ist eine deutliche Schichtung eingetreten. Man kann, wie v. Lenhossék es bei der Maus beschrieben hat, drei Strata unterscheiden.

Am weitesten nach innen liegt eine Zone grösserer Zylinderzellen, welche den späteren Haarzellen entspricht. Ihre Kerne sind bereits in die basale Partie des Zellkörpers gerückt; sie sind sämtlich durch eine grosse Zahl eingelagerter dunkler Körnchen charakterisiert.

Dann folgt ein Stratum plexiforme, in dem die eindringenden Nervenfäserchen rechtwinklig umbiegen, sich vielfach teilen und einen zu der Oberfläche des Organs parallel gerichteten Filz bilden, und drittens eine breite Zone kleiner, fast kubischer Zellen mit dunkleren Kernen, in welcher massenhafte Nervenfasern in senkrechter oder schräger Richtung verlaufen. Es galt hier festzustellen, ob bereits Nervenfasern mit Zellen der Innenzone in Beziehung treten. DerartigeVerbindungen sind vorhanden, aber nur in geringer Zahl; sie markierten sich scharf durch die eigentümliche Form und Struktur der betreffenden Zellen. Während nämlich die überwiegende Mehrzahl der Zylinderzellen ein blasses, homogenes Protoplasma aufwies, sah man hier und da etwas grössere Exemplare, welche durch eine deutliche Wabenstruktur sowohl im oberen wie im basalen Teile des Zellkörpers auffielen. Auch der Kern war an derartigen Exemplaren grösser und dunkler gefärbt, als bei denjenigen der Nachbarschaft. (cf. Fig. 19, Taf. VI). Bei genauer Betrachtung liess sich nun immer konstatieren, dass an das aussere spitze Ende einer solchen Zelle ein Nervenfäserchen herantrat, welches sich dort an ihrer Ober- 
fläche in ein zartes Fibrillennetz auflöste. In der Regel liessen sich diese ausserordentlich zarten und nicht immer leicht erkennbaren. Terminalfibrillen nur bis zum Niveau der unteren Kerngrenze nachweisen. Die Reduktionsmethode zeigt uns die Fibrillen beim Menschen hier in ähnlicher Anordnung, wie sie von Krause mit der Golgischen Imprägnationstechnik an Lachsembryonen dargestellt worden sind. Dieser Autor fand, dass die Nervenfaser nach ihrem Eintritt ins Epithel an ihrem freien Ende sich napfförmig aushöhlt und hier an das Ende einer Epithelzelle anschmiegt. Aus diesen Endnäpfen spriessen dann feinste Fibrillen hervor, welche die Zelle allseitig umspinnen. Die Veränderungen, die im Körper der Epithelzelle nach erfolgtem Kontakt mit den Neurofibrillen sich vollziehen, konnte Kra use mit der Golgischen Methode natürlich nicht feststellen.

In vollkommenem Einklang mit den beim Menschen erhobenen Befunden stehen die Bilder, welche uns die Hörbläschen in Scylliumembryonen boten. Auch hier sehen wir, dass in einem Verbande indifferenter Epithelien sich erst dann grosse Sinneszellen bilden, wenn die schrittweise vom Aussenrande der Hörbläschenwandung nach innen verfolgbaren $\mathrm{N}$ erve nfädchen mit den Zellen in Verbindung treten.

Fig. 20, Taf. VI, veranschaulicht den Moment, in welchem Zelle und Faser miteinander verschmelzen. Die intrazellulären Ringe, welche für die reife Haarzelle charakteristisch sind, fehlen in diesem Stadium der Entwicklung noch vollkommen. Unsere Befunde sprechen also dafür, dass die Verschmelzung von Faser und Zelle eine sekundäre ist. Eine höhere Differenzierung erlangt das Protoplasma der Haarzellen erst dann, wenn die Verbindung mit der von aussen kommenden Nervenfaser bergestellt ist. Lägen die Dinge umgekehrt, so müsste man erwarten, dass die Reifungserscheinungen in den Haarzellen dem Auftreten der Nervenfasern in den Endorganen vorangingen. Dass aber dies nicht der Fall ist, geht aus dem Vergleich der beiden geschilderten Entwicklungsphasen deutlich hervor.

Ein Nebenbefund darf nicht unerwähnt bleiben. Er betrifft die freien, spitzen Ausläufer der Nervenfasern an den Stellen, wo noch keine Verbindung mit den Zellen der Innenzone erreicht ist. Diese Endigungsweise is t nur eine schein- 
bare. Betrachtet man unsere Praparate von dem Embryo aus dem Ende des zweiten Schwangerschaftsmonats, dann sieht man bei Anwendung starker Blenden, dass zarte Protoplasmabrücken $z$ wischen den freien Spitzen der Nervenfäserchen und den Basalteilen der noch undifferenzierten Haarzellen in grosser Menge ausgespannt sind. Ja, man hat häufig den Eindruck, als ob ein breiter Plexus blasser Protoplasmafäden von der Haarzellenzone durch das Stratum plexiforme hindurch bis in den Bereich der Fadenzellen sich erstreckt. Die nervösen Elemente scheinen an und in diesen Brücken entlang zu ziehen. Man wird diese Beobachtung kaum anders deuten können, als dass wir es hier mit eigentümlich angeordneten Interzellularbrücken zu tun haben, welche der wachsenden Nervenfaser ibren Weg genau volzeichnen. Darin liegt eine Bestätigung der von $\mathrm{Held}$ vertretenen Anschauung, dass es freie Nervenendigungen nicht gibt, sondern dass die von bestimmten Bildungszellen ausgehenden Fibrillenleitungen den Wegen primärer oder zellig komplizierter Interzellularbrücken folgen. Wahrscheinlich spielen die aus dem primären Ganglion eindringenden $\mathrm{H}$ arris on schen Zellen, welche Lenhossék neuerdings als Lemmoblasten bezeichnet hat, bei diesem Bahnungsprozess eine wichtige Rolle. Nachzutragen wïre noch, dass die erwähnten Plasmabrïcken auch in dem von dem jüngeren Embryo stammenden Präparat sichtbar sind; nur wird hier das Bild durch die übergrosse Zahl dicht beieinander liegender Zellgebilde resp. Kerne auch an sehr dünnen Schnitten stark getrübt.

\section{Ganglion spirale und radix cochlearis.}

Viel grössere Schwierigkeiten als die Endorgane des Vestibularnerven bieten der histologischen Analyse die Endstellen des Nervus cochlearis. Denn der Verlauf der marklosen Endstrecke ist in der Schnecke ein recht komplizierter; die Form und Anordnung der Epithelien ist hier viel mannigfaltiger als in den Maculae und Cristae, und dazu kommt, dass selbst die topographischen Beziehungen dieser Zellen zueinander noch in manchen Punkten trotz hervorragender Arbeiten, unter denen die von Retzius ${ }^{1}$ ) und Held (I.c.) obenan stehen, der Klärung bedürfen.

1) Retzius: Das Gehörorgan der Wirbeltiere. Stockholm 1881 und 1884 . 
Die Bilder, welche die Silberaldehydmetbode liefert, sind wohl geeignet, unsere Kenntnisse über den Verlauf der Endstrecke des Cochlearis in manchen Punkten sicher zu stellen und zu erweitern; denn keine der bekannten Methoden liefert wohl in quantitativer Hinsicht eine so vollständige Darstellung der marklosen nervösen Elemente; aber auch unsere Präparate sind von dem erstrebenswerten Idealbilde noch entfernt, weil die Darstellung der zelligen Gebilde nicht auf gleicher Höhe steht wie die der Ner.venfasern. Auch die für die Erkenntnis der öltlichen Beziehungen der Zellen zueinander wichtigen Stützfasern kommen so gut wie gar nicht in unseren Präparaten zur Darstellung.

Wir müssen bei unserer Besprechung in Anbetracht der kaum übersehbaren Literatur darauf verzichten, alle Vorarbeiten gebührend zu würdigen, und wollen uns daher im wesentlichen auf die Beschreibung unserer Befunde beschränken Nur an einzelnen wichtigen Stellen soll auf die Anschauungen früherer Autoren eingegangen werden.

Das Übersichtsbild des Spiralganglions entspricht in unseren Präparaten ganz demjenigen, welches Zell- und Markscheidenfarbungen zeigen. Auf Radialschnitten finden wir die yon mehr oder weniger breiten Faserbündeln durchzogenen Anhäufungen bipolarer Zellen, welche einen stärkeren Fortsatz zum Gehirn und einen schwächeren zum Corti schen Organ hinsenden (Fig. 1, Taf. V). Sie gleichen im allgemeinen den Zellen des Scarpaschen Ganglions; nur sind sie etwas kleiner und von regelmässigerer, fast kugeliger Gestalt. Auch das Fibrillennetz in ihrem Zelliörper ist etwas zarter und feinmaschiger als bei jenen (Fig. 2, Taf. V). Multipolare Elemente fehlen auch hier vollkommen oder sind, wenn man sie gelegentlich findet, ohne weiteres als Kunstprodukte erkennbar, welche durch eine ungleichmässige Retraction der Zellränder von der Kapsel unter dem Einfluss der Fixierungsmittel entstanden sind. Am peripheren Pole des Ganglions kreuzen die markhaltigen Fasern auf Radialschnitten zahlreiche Spiralbündel und lassen sich dann. zwischen den Lamellen der Lamina spiralis ossea bis zur Habenula perforata verfolgen, wo sie ihre Hüllen verlieren und dann schräg aufwärts als marklose Bündelchen durch die Foramina nervina hindurch treten. An Horizontalschnitten, welche zur Basilarmembran etwas schräg 
geneigt sind, lässt sich leicht feststellen, dass beim Meerschweinchen ungefähr 6-10 Fäserchen ein solches Bündel zusammensetzen. Gleich nach ihrem Durchtritt bi e g en sie dan n alle rechtwinklig um; und gleichzeitig erfahren die meisten eine deutliche Bifurcation (Fig. 15, Taf.VI). Hier kommt also eine vollkommene Änderung in der Verlaufsrichtung zustande, indem die bisher radiär ziehenden Fasern sämtlich zu Spiralfasern werden. Die Distanz zwischen dem Nervenloch und der Umbiegungsstelle ist nicht für alle Fasern die gleiche, und dadurch kommt eine Trennung dieses innersten marklosen Spiralfaserzuges in zwei, manchmal auch drei parallel verlaufende Bündel zustande, deren Querschnittsbilder sich auch auf Radialschnitten als kreisrunde, schwarzpunktierte Felder immer scharf markieren. An der Bifurcationsstelle fällt meist auf, dass der zentralwärts zum Schneckengipfel sich wendende Fortsatz ein viel stärkeres Kaliber aufweist, als der entgegengesetzte. Dieses Verhalten erinnert an die Teilungsvorgänge der hinteren Wurzelfasern nach ihrem Eintritt in die Substanz der Hinterstränge, wo auch ein starker Ast zentralwärts zur Medula oblongata und ein schwacher in der Richtung nach der Cauda equina verfolgbar ist. Es verdient hervorgehoben zu werden, dass die Spiralfaserzüge sich aus $\mathrm{scharf}$ begrenzten, parallel gerichteten Einzelfasern zusamensetzen. Von einer Bildung, „diffuser Plexus" oder einer „spinnennetzartigen Durchflechtung" der Füserchen können wir an unseren Präparaten nichts wahrnehmen. Wir befinden uns bier im Gebiete der W aldeyerschen Körnerschicht, welche nach aussen von den inneren Pfeilern und nach oben von den Körpern der inneren Haarzellen begrenzt ist. Das zerklüftete Aussehen, welches dieser Region in den mit den gewöhnlichen Farbstoffen tingierten Präparaten eigen ist, zeigt sie auch im Fibrillenbilde. Sie kennzeichnet sich auf unseren Horizontalschnitten als ein Reticulum gröberer und feinerer Protoplasmabălkchen mit eingestreuten dunklen Kernen. Von $\mathrm{Held}$ ist darauf hingewiesen worden, dass die Balken dieser zerklüfteten Formation verschiedenen Zelltypen angehören. $\mathrm{Er}$ spricht sie als Produkte der inneren Phalangenzellen und der Grenzzellen an, die sich mit ibren weichen und am fixierten Präparat grob vakuolisierten und zersprengten Körpern den inneren Haarzellen seitlich und nach unten anschmiegen. Wir können gegen diese Darstellung $\mathrm{Helds}$ nichts 
einwenden; glauben aber mit Bestimmtheit annehmen zu können, dass das Plasma der Haarzellen an diesem Balkenwerk erheblich beteiligt ist. Wir sehen nämlich im Gegensatz zu Held, dass der Haarzellenkörper nur selten nach unten durch eine scharfe Grenzlinie abgeschlossen ist, sondern dass er sich vielmehr in eine grosse Zahl von Balken auflöst, zwischen denen mehr oder weniger grosse Lücken liegen. Dass dadurch dendritenähnliche Verzweigungen zustande kommen müssen, von denen seit Hensen viel in der Literatur die Rede ist, liegt auf der Hand. Ist demnach auf der einen Seite $\mathrm{Held}$ beizupflichten, dass sich hier unter dem Einfluss der Fixation Artefakte bilden, so scheint doch andererseits die Gesetzmässigkeit, mit der dieselben Bildungen immer wieder hervortreten, darauf hinzudeuten, dass basale Fortsätze an den Haarzellen präformiert sind, deren genaue Begrenzung gegen benachbarte Gebilde bisher aber nicht möglich war.

Was uns in dieser Gegend am meisten interessiert, ist die Frage, in welcher Weise die inneren Haarzellen innerviert werden. Betrachtet man etwas schräge Horizontalschnitte, so sieht man, dass das Protoplasmareticulum der Waldeyerschen Körnerschicht alle Spiralfaserzüge einschliesst und zwischen die einzelnen Bündel derselben eindringt. Manchmal hat es den Anschein, als ob die Haarzellen mit ibren zerklüfteten Fussformationen auf den Spiralfaserzügen mit breiter Basis aufsitzen. In diese Berübrungszone sieht man nun radiärverlaufende Nervenelemente nach innen und oben zum Haarzellenkörper aufsteigen, welche sich nach Art von Collateralen aus einer längeren Spiralfaser entwickeln(Fig. 15̆, Taf.VI). Gesetzmässigkeiten in der Verlaufsrichtung lassen sich nicht feststellen. Es kommen vielmehr verschiedene Modalitaten vor. Manchmal findet man, dass diese aufsteigenden Ästchen nach vorher gegangener gabeliger Teilung mit zwei spitzen kurzen Ausläufern den unteren Teil der Zelle berühren (Fig. 16, Taf. VI), manchmal sieht man Schlingenformationen, welche aus dem zunächst gelegenen Spiralbündel aufsteigen und wieder zu ihm zurückkehren (Fig. 17, Taf. VI). Derartige schmale Schlingen reichen nicht selten bis in das Kernniveau der Haarzellen hinauf. Auf Radialschnitten sieht man schliesslich, dass der basale Teil der Zellen mitunter von einem ganzen Netzwerk feinster Nervenfibrillen umsponnen wird, welche 
miteinander anastomosieren (Fig. 18, Taf. VI). Diese Art der Innervation ist die räumlich extensivste, lässt sich aber auch in gut gelungenen Präparaten nur selten nachweisen, und es ist deshalb fraglich, ob die beiden vorher geschilderten Arten nur als Ausdruck einer Teilimprägnation zu betrachten sind. Intrazelluläre Nervenendigungen haben wir mit Sicherheit nie feststellen können, wenngleich zugegeben werden muss, dass bei dem geringen Breitendurchmesser der Sinneszellen eine genaue Bestimmung von ,,innen" und ,aussen" schwierig ist. Es ist an dieser Stelle noch hervorzuheben, dass irgend ein Zusammenhang zwischen den aus den Foramina nervina hervortretenden Cochlearisfasern und den Zellen der Körnerschicht $\mathrm{n}$ ic $\mathrm{h}$ t besteht. Waldeyer und Gottste in betrachteten diese Zellen als Teilerscheinung eines Nervenapparates. Sie brachten die Zellausläufer mit den nervösen Elementen in kontinuierlichen Zusammenhang und vergleichen sie mit der inneren Körnerschicht der Retina. Nachdem Retzius diese Ansicht widerlegt hatte, ist sie fast gänzlich verlassen worden. Neuerdings hat aber Ichita $\mathrm{Kishi}{ }^{1}$ ) diese Lehre auf Grund von Fürbungen mit dem Apathyschen Hämateïn neu zu stïtzen yersucht, indem er zu sehen glaubte, dass die Zellen der fraglichen Region gewissermassen in den Verlauf der Nervenfasern eingeschaltet seien. Diese Anschauung erscheint uns auf Grund unserer Bilder als unwahrscheinlich; denn niemals lässt sich auch nur ein oberflachlicher Kontakt $z$ wischen den Neurofibrillen und jenen Zellen erkennen. Über die Natur und Herkunft der inneren Körner geben unsere Präparate keine Auskunft; docb scheint die Darstellung von Retzius, wonach sie zum indifferenten Epithel gehören, am meisten den Tatsachen zu entsprechen. $\mathrm{Hel} \mathrm{d}$ hat in der erwähnten Arbeit diese Anschauungen durch eine detaillierte Beschreibung noch weiter begründet. Die Ansicht Schwalbes, dass die Kerne zu den Nerven vielleicht als Kerne von Gliazellen gehören, ähnlich denen, welche sich in der marklosen Nervenfaserschicht der Retina finden, hat nach unseren Bildern nicht viel für sich, weil die räumliche Distanz zwischen beiden Elementen eine zu grosse ist.

1) I c hita Kishi: Über den peripherischen Verlauf und die Endigungen des Nervus chochlearis. Arch. f. mikr. Anatomie und Entwicklungsgeschichte, Bd. 95, 1901. 
Aus den beschriebenen inneren Spiralfaserzügen entwickeln sich als Seitenäste nach Art von Collateralen in ziemlich regelmässigen Abständen radiär gerichtete Fasern, welche in der bekannten Weise in den Tunnelraum eindringen, denselben durchziehen und ihn durch die Lücken zwischen den Aussenpfeilern wieder verlassen. Dabei sind $z$ wei Momente beachtenswert, durch welche sich unsere Befunde von der Mehrzahl der früheren Darstellungen unterscheiden.

Erstens sehen wir niemals Spiral faserzüge im Tunnelraum selbst. Nachdem sich aus den inneren Spiralfaserzügen im Bereich der Körnerschicht radiäre Fasern formiert haben, behalten diese die radiäre Richtung zunächst fest und lagern sich im Tunnelraum nicht wieder um. Das zeigen nicht bloss unsere Präparate von Meerschweinchen, sondern auch solche von höherstehenden Säugern, wie z. B. von Macacus und Cercopithecus. Am besten lassen sich diese Verhältnisse an Horizontalabschnitten beurteilen, wie z. B Fig. 21 einen von der Meerkatze (Cercopithecus sabaeus) darstellt. Hier ist von einer longitudinalen Umbiegung der in radiürer Richtung den Tunnelraum betretenden Elemente keine Rede. Die Punktformationen im inneren und äusseren unteren Winkel des Tunnels, welche von den Autoren seit Retzius an den üblichen Radialschnitten als quergetroffene Spiralzüge gedeutet worden sind, verlangen nach unserer Ansicht eine andere Erklärung. Es handelt sich hier um granulierte Plasmaanhäufungen, welche an Teilungsstellen markloser Fäserchen häufig gefunden werden; und hier finden solche Bifurcationen, wie gleich erörtert werden wird, in grosser Zahl statt. Dass übrigens gelegentlich einmal ein in den Tunnelraum eindringendes Radialfäserchen einen kurzen Umweg an der Innenwand des Tunnels macht, wollen wir nicht in Abrede stellen; um ein gesetzmässiges Vorkommen handelt es sich dabei aber unseres Erachtens nicht. Die Golgiimprägnationen, welche bisher für das Studium der marklosen Strecken des Cochlearnerven die besten Resultate lieferten, führen gerade an dieser Stelle leicht zu Irrtümern. Man sieht nämlich, auch wenn die Fasern gut gefarrbt sind, die benachbarten Zellgebilde niemals ganz deutlich und weiss deshalb nicht recht, wo man sich befindet. Auf diese Weise werden Fasern, welche zu den Spiralzügen der Körnerschicht gehören, leicht in den Tunnelraum verlegt. Dazu kommt, dass die räumlichen Verhältnisse am embryonalen Material, welches 
hier für die Golgische Methode allein in Betracht kommt, viel schwerer zu beurteilen sind, als an reifen Objekten.

Der zweite Punkt, welcher eine gewisse Bedeutung verdient, ist der, dass die Fibrillenbilder auf Radialschnitten sehr häufig zwei radiäre Tunnelfasern aufweisen, von denen die eine den Raum in mittlerer Höhe, die andere am Boden oder in geringer Entfernung von demselben durchzieht (Fig. 11, Taf. VI). Manchmal kommt auch noch eine dritte Radiärfaser (Fig. 12, Taf. VI) vor. Es gelingt nicht selten, die Einzelfaser bis zu ihrem spiralen Ursprungsbündel in der Körnerschicht $z u$ verfolgen. Dann sieht man, dass die oberen und unteren Fasern auch aus verschiedenen Spiralbündeln hervorgehen, meist in der Weise, dass die unteren mit den unteren, die oberen mit den oberen Spiralbündeln zusammenhängen (cf. Fig. 13, Taf. VI). Sehr deutlich illustriert dieses Verhalten auch Fig. 14, Taf. VI, in der man sehen kann, dass die Tunnelfasern aus verschiedenen Quellen ihre Fibrillen beziehen, und dass diese sich manchmal erst nach einer längeren Strecke isolierten Verlaufes zu einem starkkalibrigen Fibrillenbündel vereinigen. Von den tropfenförmigen Varikositäten, welche man im Golgibilde und an gefärbten Schnitten sehr bäufig findet, sind auch im Fibrillenbilde nicht selten Andeutungen vorhanden, welche sich als rundliche „perifibrilläre Plasmaanhäufungen" präsentieren. Wahrscheinlich handelt es sich hierbei um kadaveröse Bildungen, welche um so häufiger sind, je später das Untersuchungsmaterial fixiert wird.

Nachdem die Radiärfasern den Tunnelraum verlassen und den $\mathrm{Nu}$ ëlschen Raum durchquert haben, teilen sie sich in mehrere kleine Ästchen und streben dem oberen und mittleren Teil der zunachst gelegenen Deitersschen Zelle zu, um scheinbar an ihrer dem Tunnelraum zugewandten Oberfläche $\mathrm{zu}$ inserieren. Dabei ist hervorzuheben, dass die Ausläufer der oberen und unteren Fasern hier zusammenstossen und in einer gemeinsamen Zone am Rande der Zelle miteinander verschmelzen Dieses Insertionsgebiet der Radiärfasern an den Deitersschen Zellen hat im Fibrillenbilde ein ganz eigentümliches Gepräge. Es hat die Gestalt eines mehr oder weniger breiten streifens, der sich in der Regel ziemlich scharf von der anstossenden Stützzelle abgrenzen lässt. Wir wollen ihn als „nervösen Archiv f. mikrosk. Anat. Bd. 71. 
Randstreifen " bezeichnen. An seiner Bildung sind Fiorillen und Axoplasma in gleicher Weise beteiligt; man erkennt dies daran, dass die zarten, schwarzen Nervenfädchen in eine durch Goldchlorid rötlich tingierte, homogene Grundmasse eingebettet sind, welche man bis in dje Teilungswinkel der Stammfaser hinein verfolgen kann. In diesen Randstreifen tauchen, wie schon erwähnt wurde, ausser den oberen Tunnelfasern auch von unten her die Fibrillen der unteren Tunnelfasern ein. Einen derartigen Streifen sehen wir auf Radialschnitten an jeder Deitersschen Zelle auf der dem Tunnel zugewandten Seite. Er ist in der Regel am breitesten an der ersten dem Tunnel zunächst gelegenen, am schmalsten in der dritten, am weitesten von ihm entfernten Zelle. Auch seine Länge schwankt in breiten Grenzen; manchmal reicht er fast bis zur Basilarmembran, manchmal nur bis zur Kernhöhe der Deitersschen Zelle. Die Innervation des Streifens in der zweiten und dritten Reihe erfolgt in ganz ähnlicher Weise wie in der ersten, nämlich durch Radiärfasern, welche direkt aus den inneren Spiralfaserzügen der Körnerschicht dorthin verfolgbar sind (cf. Fig. 12, Taf. VI) oder durch Seitenäste solcher Fäserchen, welche die Formierung des ersten Streifens besorgen (Fig. 13, Taf. VI).

Der Verlauf der Fibrillen in den einzelnen Randstreifen ist ein sehr variabler. Auf Radialschnitten findet man vertikal gerichtete Fädchen neben horizontalen Elementen, deren Querschnitte als Punktreiben mit mehr oder minder grossen Intervallen erscheinen.

Es kann keinem $\mathrm{Z}$ weifel unterliegen, dass der $\mathrm{R}$ a $\mathrm{n} d \mathrm{~s} \mathrm{t} \mathrm{r}$ e if e $\mathrm{n}$ mit den äusseren Spiralfaserzügen der Autoren identisch ist; denn tatsächlich verlaufen hier, wie man am besten an Horizontalschnitten sieht, viel spiralige Elemente, welche aus einer direkten Umbiegung der Radiärfibrillen der Tunnelfasern hervorgehen. Die Länge dieser Spiralfibrillen scheint bei verschiedenen Tierarten verschieden $\mathrm{zu}$ sein; beim Meerschweinchen waren sie jedenfalls viel kürzer wie bei den untersuchten Affen, wo man sie gelegentlich an fünf und noch mehr Zellen vorbeiziehen sehen konnte, um an entsprechender Stelle an einer entfernten Zelle scheinbar blind zu endigen. Aber diese Spiralfasern bilden, wie bereits erwähnt, nur einen Teil des fibrillären Inhaltes der Randstreifen. Denn vertikal und schräg verlaufende Fibrillen 
sind manchmal in so grosser Zahl vorhanden, dass sie jene vollkommen verdecken. Dazu kommt, dass sich in der fraglichen Zone sehr viele Fäserchen teilen, sodass man an den am meisten gefärbten Präparaten den Eindruck eines Fibrillenfilzes hat, in dessen Maschen eine ziemlich dunkle Plasmamasse liegt. Ganz eigenartige Bilder erscheinen da, wo die Eintrittsstellen der oberen und unteren Tunnelfasern in den Randstreifen mit seinem oberen und unteren Ende zusammenfallen. Dann entstehen Figuren, welche man mit grossen Schleifen vergleichen kann, deren Schenkel von den beiden Tunnelfasern gebildet werden, und deren nach aussen gelegener Pol eben jener Randstreifen an der Deitersschen Zelle ist.

Man wird hier an die Fibrillenschleifen in den Meissnerscben Tastkörperchen und in anderen sensiblen Endorganen del Haut erinnert, wo ebenfalls die marklos gewordenen Nervenfäserchen büscheiförmig auseinander weichen, um sich dann wieder $z u$ einem homogenen Nervenfäserchen $z u$ vereinigen und in die Stammfaser zurückkehren. Diese Vergleichung ist a uch insofern zutreffend, als hier wie dort in demjenigen Gebiete, wo die Fibrillen auseinander weichen, eine absolute Zunahme sowohl der fibrillaren wie der interfibrillären Plasmasubstanzdes Achsenzylinders stattfindet. Bei dieser Betrachtung der Dinge muss man demnach den Randstreifen als die letzte Endigung der Cochlearisfasern im Bereich der äusseren Haarzellen auffassen.

In welcher Verbindung stebt nun aber dieses Gebilde mit den perzipierenden Haarzellen? Eine solche Verbindung muss ja unbedingt vorhanden sein, wenn die herrschende Auffassung von der Funktion dieser Zellen richtig ist. Betrachtet man die Abbildungen unserer Präparate, so sieht man übereinstimmend, dass das obere Ende des Randstreifens bis in das Niveau der unteren Harzellengrenze hinaufreicht, ja dass sie diese auch noch stellenweise überragt. Hier erfolgt eine Verschmelzung des interfibrillären Axoplasmas mit dem Protoplasma der Haarzellen. Man kann das am besten an Prăparaten feststellen, in denen die Fibrillen des Randstreifens unvollständig gefärbt sind. Dort sieht dann seine plasmatische Substanz wie ein nach unten und vorn gerichteter Fortsatz der Haarzelle aus, und eine scharfe Grenze zwischen 
Zellplasma und Axoplasma ist nicht zu erkennen. Dass auch die Fibrillen des Randstreifens mit dem unteren Haarzellenpol innig verwachsen sein müssen, ergibt sich aus dieser Darstellung von selbst.

Unsere Bilder lassen sich auch mit den Beschreibungen derjenigen Autoren, welche die Nervenendstellen an den äusseren Haarzellen am eingehendsten untersucht haben, $\mathrm{Katz}^{1}$ ) und $\mathrm{Held}^{2}$ ) in vollen Einklang bringen. Katz gibt an, dass der untere Teil der Cortischen Zelle von der in der Deitersschen Zelle gelegenen Stützfaser, wie von einem "Zangenbecher" umfasst wird. Dieser Zangenbecher soll einen nach der Seite der ausseren Tunnelfaser offenen Schlitz haben, durch welchen die Endästchen nervöser Fasern eintreten und ein kelchähnliches Ende zeigen. Auf Zupfpräparaten erscheint dasselbe als eine grob granulierte Masse am unteren Haarzellenende und füllt den Raum zwischen der Cortischen Zelle und dem zangenbecherförmigen Gebilde aus. Held, der die topographischen Beziehungen $z$ wischen den Haar- und Deitersschen Zellen bis in die feinsten Details erschlossen hat, konnte mit Hilfe seiner Neurosomenfärbung die Angaben von Katz bestätigen. Auch er beschreibt einen Nervenraum an der basalen Grenze der äusseren Haarzellen, in welchen die Terminalfäserchen als fein granulierte Fädchen eintreten; hier sollen sie zu einer ungefähr kegelförmigen dunkelgefürbten Masse verschmelzen, welche sich aus einer Summe feinster Fäserchen und sogenannten Endfüssen zusammensetzt. Ein ăhnliches Verhalten hat wohl auch $\mathrm{K}$ is hi beobachtet, welcher am unteren Ende der Haarzellen Gebilde von kelchförmiger Gestalt gesehen hat, welche durch kurze Fädchen mit den äusseren Spiralnervenzügen verbunden sind. Er betrach tet diese Kelchgebilde als Teile der Haarzelle selbst, obwobl sie in seinen Abbildungen durch eine Membran von der Substanz der Zelle getrennt zu sein scheinen.

$\mathrm{Nach}$ unserer Ansicht sind die nervösen Endgebilde der genannten Autoren mit dem am weitesten nach oben gelegenen Teile unseres Randstreifens identisch. Dass sie in der Darstellung jener Autoren als besondere, von dem übrigen Teile des Rand-

1) Katz: Über die Endigung des Nervus cochleae im Cortischen Organ. Arch. f. Ohrenheilkunde. Bd. 29. 1890.

2) Held: l. c. 
streifens getrennte Gebilde imponieren, ist durch die Eigenart ihrer Präparate, in denen die Retziusschen Stützfasern gleichzeitig mit den nervösen Elementen zu Gesichte kommen, bedingt. Die Anordnung der Stïtzfasern bringt es mit sich, dass der an der Berührungsstelle mit der Haarzelle gelegene Pol des Randstreifens als ein von dem übrigen Teile getrenntes Gebilde erscheint. Sieht man sich die Abbildungen von Held genauer an, so z. B. seine Fig. 29 und 37, so kann man auch an ihnen Belege für die Richtigkeit unserer Auffassung entnehmen. Denn an der dem Tunnel zugewandten Oberfläche der D eit e rsschen Zellen liegen seine Neurosomenhäufchen in sehr dichter Anordnung, fast ebenso dicht wie in den Endfüssen innerhalb der Nervenräume. Ja man hat den Eindruck, dass diese an den Deitersschen Zellen gelegenen Neurosomengruppen zu ähnlichen Netzfiguren miteinander verbunden sind, wie man sie an den Fibrillen unserer Randstreifen findet.

Auch die Darstellung, welche Retzius über die Beziehungen der äusseren Spiralfasern zum Basalpol der Haarzellen gibt, steht mit unseren Befunden zum mindesten nicht in Widerspruch. Die obersten Spiralfäserchen ziehen nach seiner Meinung nicht nach den Zellen empor, sondern kommen mit deren unteren Enden direkt in Berührung. An diesen Zellenenden liegen glänzende Körper, welche an den Nervenfasern haften und die möglicherweise eine Vermittlerrolle zwischen beiden spielen. Held identifiziert die glänzenden Körperchen mit seinen Neurosomenhaufen. Der oberste Zug der Spiralfasern von Retzius und seine Körperchen am Pol der Haarzellen liegen im obersten Bereich unseres Randstreifens und sind bereits als Teile der Nervenendstelle zu betrachten, sodass nach einer besonderen Endformation, welche Retzius noch vermisst, unseres Erachtens nicht mehr gesucht zu werden braucht.

Dass unsere Randstreifen auch von anderen Autoren gesehen, nur in ihrer Bedeutung nicht erkannt worden sind, das lehrt auch eine Abbildung, welche Ebner im Koellikerschen Handbuch der Gewebelehre des Menschen ${ }^{1}$ ) gibt. Hier sieht man an einem radialen Durchschnitt durch das Cortische Organ vom unteren Rande der äusseren Haarzellen breite, granulierte Streifen basalwărts ziehen. In

1) Leipzig, 1902, 6. Aufl., pag. 935, Fig. 14557. 
den zur ersten Haarzelle gehörigen Streifen treten, wie auf unseren Präparaten, sowohl obere wie untere Tunnelfasern ein. Ebner bezeichnet die fraglichen Stellen als die drei Spiralnervengeflechte unter den Haarzellen. Man hat jedoch vielmehr den Eindruck, dass es sich hier um breite Plasmabander handelt, in welche die Fibrillen der $\mathrm{R}$ adialfasern sich in irgend einer allerdings nicht näher erkennbaren Weise auflösen. Eine Abweichung von unseren Bildern besteht nur darin, dass eine deutliche Grenze zwischen dem unteren Zellrand und dem Plasmastreifen vorhanden ist, welche wir nicht sehen.

Sind nun die geschilderten Verhältnisse als Ausdruck eines blossen Kontaktes oder einer kontinuierlichen Verbindung zwischen Nerv- und Sinneszelle zu betrachten? Die Frage ist ungleich schwieriger zu beantworten als an den Endstellen des Vestibularnerven, weil in der Schnecke die Berührungsfläche zwischen beiden Gebilden viel kleiner und optisch schwerer analysierbar ist. Immerhin glauben wir sagen zu können, dass man mit der Vorstellung eines blossen Kontaktes den Tatsachen nicht gerecht wird. Die Verschmelzung des Nervenendprotoplasmas mit dem Zellprotoplasma ist eine so innige, dass eine klare Grenze überhaupt nicht mehr zu Tage tritt, und deshalb muss man Held beipflichten, wenn er von einer Verwachsung der Hörnervenfäserchen mit dem basalen Zellabschnitte spricht. In der Aussenschicht dieser Verwachsungszone bilden die letzten Ausläufer der Neurofibrillen netzartige und schleifenförmige Figuren, ohne dass ein Eindringen derselben in den Zellkörper jemals sicher von uns beobachtet wurde. Es fehlen hier auch die Ringfolmationen, welche wir am Boden der Haarzellen in den Maculae und Cristae acusticae fanden, vollkommen.

Dass auch hier diese Konkreszenz eine sekundäre ist, wird man schon nach Analogie der embryologischen Befunde an den Maculae und Cristae annebmen dürfen. Wir verfügen auch über eine Reihe von Präparaten aus frühen Entwicklungsstadien der Schneckengänge, in denen man das schrittweise Vordringen der Nervenfasern vom Acusticusganglion in das Epithel der Windungen verfolgen kaun. Unsere Befunde sind aber nach dieser Richtung noch unvollständig und sollen späterhin ergănzt werden.

Mit einigen Worten wäre noch auf die Struktur der ă us s e r e n Haarzellen einzugehen, welche in unseren Fibrillenbildern ein 
eigenartiges Gepräge besitzen. Man kann drei Zonen in derselben unterscheiden.

Die oberste dorsale, welche unter der Deckplatte gelegen ist, enthalt grobe Wabenstrukturen und fällt schon bei schwacher Vergrösserung durch ihr runzeliges Aussehen auf. Die mittlere bildet ein belles Bläschen, an dessen Boden sich der Kern befindet.

Am weitesten nach unten folgt dann ein homogenes Stück, das sich basalwärts kontinuierlich in den Randstreifen fortsetzt. In dem obersten, wabigen Bezirk sieht man zuweilen einen sich von der umgebenden Protoplasmamasse scharf abhebenden knäuelförmigen Körper. Er setzt sich aus Fadchen zusammen, welche ganz die Farbe und das Aussehen zarter Neurofibrillen haben (cf. Fig. 11, Taf. VI, bei K). Der Befund musste uns stutzig machen und die Vermutung nahelegen, dass es sich hier um intrazelluläre Fibrillenauslaufer handle. Diese Annahme verlor aber viel an Wahrscheinlichkeit, als wir im Laufe unserer Untersuchungen sahen, dass sich niemals Verbindungsbrücken zwischen den Fadchen des Knäuelkörpers und den Fibrillen des Randstreifens fanden, und dass dieser Körper selbst sich nur relativ selten nachweisen liess. Seine Bedeutung ist uns auch heute noch rätselhaft. Seiner Lage nach ist er mit dem Hensenschen Körper identisch, welcher von seinem Entdecker als eine Kapsel mit dünner Wandung und spiraliger Streifung beschrieben wurde. Die Streifung schien ihm von einem die Wand der Kapsel aussen umziehenden Faden herzurühren, welcher nervöser Natur sein könne.

Sehr problematisch ist auch nach unseren Bildern noch der Zusammenhang zwischen den Randstreifen und den Deitersschen Zellen. Hier muss sich bei vorurteilsloser Betrachtung der Zweifel regen, ob die nahen örtlichen Beziehungen zu den Neurofibrillen nicht einen Hinweis für die Funktion dieser Zellen enthalten. Die in den Randstreifen entbaltenen Fibrillen berühren die Oberfläche dieser Deitersschen Zellen so innig, wie nur irgendwo Sinneszellen in Go g i bildern von nervösen Endfäserchen berührt werden (Fig. 12, Taf. VI). Dazu kommt das Vorhandensein der mit Eisenhämatoxylin fărbbaren Körnerhaufen in den Zellen selbst, welche Retzius ${ }^{1}$ ) zuerst gesehen hat. Die Lage dieser

1) Retz i u s: Zur Kenntnis der Gehörschnecke. Biol. Untersuchungen, N. F., Bd. IX. Stockholm 1900. 
Körner in der Nachbarschaft unserer Randstreifen muss den Verdacht erwecken, dass es sich hier gleichfalls um nervöse Protoplasmaelemente handelt.

Unter diesen Umständen ist es nicht unwahrscheinlich, dass die jetzt herrschende Auffassung, welche in den Deitersschen Zellen nur Stützzellen sieht, noch einmal eine Revision erfahren wird, im Sinne der alten Anschauungen von Gottstein und Waldeyer. Die Lehre, dass die Cortischen und Deitersschen Zellen als zweikernige Zwillingsgebilde zusammengehören, ist $\mathrm{zwar}$ vom histologischen Standpunkte als wideriegt $\mathrm{zu}$ betrachten; nach der Art und Weise aber, wie die letzten Nervenausläufer im Bilde unserer Färbungen sich mit beiden Zellarten in Verbindung setzen, steht die physiologische Seite der Frage nach unserer Ansicht auch fernerhin noch zur Diskussion.

Besseren Methoden wird es vorbehalten bleiben, in diesem Punkte die erwünschte Klarheit zu bringen!

\section{Erklärung der Abbildungen auf Tafel V und VI.}

Fig. 1. Ausschnitt aus einem Spiralganglion vom Meerschweinchen bei radialer Schnittführung. Obj. Zeiss' Apochrom. 8 mm, 0k. 3.

Fig. 2. Zellen aus demselben Ganglion bei stärkerer Vergrösserung. Obj. Zeiss' Apochrom. Immers. 2 mm, Komp.-Ok. 8.

Fig. 3. Zellen aus einem Scarpaschen Ganglion vom Meerschweinchen. Obj. Zeiss' Apochrom. Immers. $2 \mathrm{~mm}$, Komp.-Ok. 8.

Fig. 4. Übersichtsbild eines Querschnittes durch eine Crista ac. vom Meerschweinchen. Obj. Zeiss' Apochrom. $8 \mathrm{~mm}$, Ok. 3.

Fig. 5. Benachbarte Zellen aus einer Crista ac. Perizelluläre Auflösung von Endfibrillen. Intrazelluläre Ringformationen. Freie schlingenförmige Nervenendausläufer. Obj. Zeiss' A pochrom. Immers. $2 \mathrm{~mm}$, Komp.-Ok. 8.

Fig. 6. Sinneszellen aus einer Mac. acust. Zwei Zellen auf der linken Seite, welche ohne nervösen Endapparat gezeichnet sind, zeigen die Strukturverhältnisse im Protoplasma ihrer Zellkörper. Vergx. wie bei Fig. 5 .

Fig. 6a. "Rirge" in den Sinneszellen. Vergr. wie bej Fig. $\check{\text { o. }}$

Fig. 7. Zwei Haarzellen aus einer Crista ac., die eine mit dichtem perizellulärem Fibrillennet $z$, die andere ohne nervösen Endapparat. Veryr. wie bei Fig. 5 . 
Die Endorgane im häutigen Labyrinth der Säugetiere.

Fig. 8. Hörbläschen eines menschlichen Embryos (von $10 \mathrm{~mm}$ Steissnackenlänge) mit anliegendem Ganglion ac. Zeiss' Apochrom. $8 \mathrm{~mm}$, Ok. 4 .

Fig. 9. Die mit a bezeichnete Stelle aus Fig. 8 bei starker Vergr. Nervenfasern im Epithel mit scheinbar freien Ausläufern. Zeiss' Immers. $2 \mathrm{~mm}$, Ok. 4.

Fig. 10. Hörbläschen eines menschlichen Embryos (von $15 \mathrm{~mm}$ Steissnackenlänge). Zwei Cristae acust. mit Nervenbündeln, welche vom Gangl. vest. her verfolgbar sind. Apochrom. 8, Ok. 4.

Fig. 11-14. Radialschnitte durch das Cortische Organ vom Meerschweinchen. Man sieht die genaue Lage der Spiralfasern in der Waldeyerschen Körnerschicht, verschiedene Modifikationen im Verlaufe der radiären Tunnelfasern und die "Randstreifen" am Fuss der äusseren Haarzellen. Vergr. Zeiss' Apochrom. Immers. 2 mm, Komp.-Ok. 8.

Fig. 15. Schräg geneigter Horizontalschnitt durch die Körnerschicht von Meerschweinchen. Die Richtung des Schnittes geht etwas schräg nach oben. Man sieht die durch die Foram. nerv. durchtretenden Radiärfasern, ihre Formierung zu Spiralfaserzïgen und die Bifurkationen. Die inneren Haarzellen ruhen scheinbar auf diesen Zügen. Vergr. wie vorher.

Fig. 16-18. Verschiedene Endformationen von Neurofibrillen an der Oberfläche innerer Haarzellen. Vergr. wie oben.

Fig. 19. Herantreten einer Vestibularisfaser an den Boden einer Sinneszelle in der Crista ac. eines menschlichen Fötus (Steissnackenl. $15 \mathrm{~mm}$ ), Metamorphose des Zellkörper's. Vergr. Zeiss' Apochrom. Immers. $2 \mathrm{~mm}$, Komp.-Ok. 12.

Fig. 20. Sinnesepithel aus der Mac. ac. eines Embryo ron Scyllium can. (Länge $20 \mathrm{~mm}$ ). Vergr. wie bei Fig. 19.

Fig. 21. Horizontalschnitt durch das Cortische Organ eines erwachsenen Cercopithecus sab. Man kann die ganze marklose Strecke der Cochlearnerven von den Foram. nerv. bis zu den äusseren Spiralzügen übersehen. 